\title{
Incompetência istmocervical: cerclagem de emergência versus conduta expectante
}

\author{
Istmocervical incompetence: emergency cerclage versus expectant conduct \\ Incompetencia istmocervical: cerclaje de emergencia versus conducta expectante
}

Sabrina Cabral Rêgo ${ }^{1 *}$, Adelaide Gomes Martins ${ }^{1}$, Francisca Amanda de Souza Mesquita1.

\section{RESUMO}

Objetivo: Descrever e revisar sobre incompetência istmocervical: cerclagem de emergência versus conduta expectante. Revisão bibliográfica: Estudos anteriores indicaram que existe benefício quanto ao tratamento cirúrgico, uma vez que gestantes têm maior período de latência até o parto, maior idade gestacional ao nascimento e diminuição de taxa de prematuridade. Mulheres acometidas de cerclagem cervical para o tratamento da incompetência istmo cervical (IIC) continuam a possuir risco elevado para prematuridade. Já na cerclagem de emergência há maiores taxas de prematuridade e de maiores complicações se correlacionada à cerclagem profilática ou terapêutica. De maneira geral, a cerclagem aumenta o risco de infecções puerperais $(6 \%)$, internações hospitalares e realização de cesarianas. Considerações finais: 0 diagnóstico de incompetência cervical embasa-se, primariamente, na história clínica, podendo ser efetuado antes ou durante a gestação. A cerclagem é a conduta com maior aceitabilidade, de acordo com a literatura, devido aos índices de sobrevivência fetal e das gestantes portadoras de IIC no período que antecede o parto e após passar pelo tratamento e procedimento cirúrgico.

Palavras-chave: Gestantes, Incompetência cervical uterina, Conduta expectante.

\section{ABSTRACT}

Objective: To describe and review of ischemocervical incompetence: emergency cerclage versus expectant conduct. Bibliographic review: Previous studies have indicated that there is a benefit in terms of surgical treatment, since pregnant women have a longer latency period until delivery, a higher gestational age at birth and a lower rate of birth. prematurity. Women affected by cervical cerclage for the treatment of cervical isthmus incompetence (IIC) continue to be at high risk for prematurity. In emergency cerclage, however, there are higher rates of prematurity and greater complications if correlated with prophylactic or therapeutic cerclage. And, in general, cerclage increases the risk of puerperal infections $(6 \%)$, hospital admissions, and hospitalization. cesarean sections. Final considerations: The diagnosis of cervical incompetence is based primarily on the clinical history and can be performed before or during pregnancy. Cerclage is the most accepted procedure according to the literature, due to the rates of fetal survival and of pregnant women with IIC in the period before childbirth and after undergoing treatment and surgical procedure.

Keywords: Pregnancy, Uterine cervical incompetence, Watchful waiting.

\section{RESUMEN}

Objetivo: Describir y revisar la incompetencia istmocervical: cerclaje de emergencia versus conducta expectante. Revisión bibliográfica: Estudios prévios han indicado que existe un beneficio en términos de tratamiento quirúrgico, ya que las mujeres embarazadas tienen un período de latencia más largo hasta el parto, una mayor edad gestacional al nacer y una menor tasa de nacimientos prematuros. Las mujeres afectadas por cerclaje cervical para el tratamiento de la incompetencia del istmo cervical (IIC) continúan teniendo un alto riesgo de prematuridad. Sin embargo, en el cerclaje de emergencia, hay tasas más altas de prematuridad y mayores complicaciones si se correlacionan con el cerclaje profiláctico o terapéutico y, en general, el cerclaje aumenta el riesgo de infecciones puerperales (6\%), ingresos hospitalarios y hospitalización

1 Universidade Federal do Amazonas (UFAM), Manaus - AM.*E-mail: drasabrinarc@gmail.com 
cesáreas. Consideraciones finales: El diagnóstico de incompetencia cervical se basa principalmente en la historia clínica y puede realizarse antes o durante el embarazo. El cerclaje es el procedimiento más aceptado según la literatura, debido a las tasas de supervivencia fetal y de mujeres embarazadas con IIC en el período anterior al parto y después de someterse a tratamiento y procedimiento quirúrgico.

Palabras clave: Embarazo, Incompetencia del cuello del útero, Espera vigilante.

\section{INTRODUÇÃO}

A literatura aborda que a incompetência istmo cervical (IIC) é uma doença comumente encontrada em mulheres com idade mais avançada, podendo também ser diagnosticada em mulheres jovens, entre 15 e 19 anos. A IIC, ou mesmo insuficiência, foi abordada pela primeira vez em meados do século XVII, no ano de 1658, como a parte que o orifício do útero não seria capaz de ter a contração adequada para manter o feto dentro da cavidade do útero (GOULDING E e LIM B, 2014).

A IIC distingue como uma fraqueza congênita no ligamento do orifício interno cervical e o da parte inferior agrega ao esvaecimento e à cérvico-dilatação sem provocar dores no segundo trimestre, podendo atingir protrusão ou rotura das membranas fetais, e parto pré-termo, desenvolvendo aborto ou perda do feto em muitas momentos conseguindo prevenir (GOMES ALFM, et al., 2012). De acordo com Mattar R, et al. (1999), é uma patologia em que há uma deficiência funcional do esfíncter uterino, impedindo-o de manter-se fechado até o final da gravidez e originando perdas gestacionais periódicas na forma de abortos ou partos prematuros. É causa importante e frequente de abortamento habitual, caracterizado pela incompetência do sistema oclusivo do útero, o que possibilita a abertura do orifício interno (BRASIL, 2010).

Conforme relata Gomes ALFM, et al. (2012) a IIC classifica-se em primária, secundária e funcional. A primeira está associada ao distúrbio congênito relacionado a anormalidades dos úteros. A segunda é desenvolvida por meio de sequelas e trauma na região istmo-cervical, ocasionada por dilatações impróprias do colo, e, por fim, a funcional ocorre fora do período gestacional, quando não evidencia os defeitos no istmo, conseguindo ser perceptível após a gravidez. A cerclagem de emergência proporciona maiores taxas em relação à prematuridade e ainda de complicações quando comparada com a cerclagem profilática ou terapêutica (KHAN MJ, et al., 2012). Os resultados neonatais também conseguem ser apresentados de forma desfavorável na cerclagem de emergência, mesmo quando são avaliados apenas recém-nascidos que constituem baixo peso, dando a entender que exista uma influência de processos inflamatórios subclínicos nesses resultados (KUON RJ, et al., 2015).

A IIC não tem um valor etiológico bem especificado, mas pode dar sinais de alguns fatores de risco que pode ocasionar. Em outros casos, sua maior frequência ocorre após procedimentos cirúrgicos, ou a conização uterina, cirurgia de dilatação mecânica do útero e de alta frequência. Segundo American College of Obstetrics and Gynecologist (ACOG) (2014), alguns fatores que causam risco de IIC estão correlacionados a malformações mullerianas, a deficiência de colágeno e elastina no colo, além da exposição intrauterina ao dietilestilbestrol. Normalmente, a Incompetência istmo-cervical não gera sintomas, é suspeitada após uma ou mais perdas sem motivo aparente, não há contração, dor ou inflamação (CARVALHO EM, 2010). Se diagnosticado no início o tratamento da IIC, pode salvar o feto e prevenir as suas recidivas.

O diagnóstico de incompetência cervical deve ser analisado inicialmente pelo histórico clínico, podendo ser obtido antes ou durante a gestação. Depois de levantado o histórico clínico, deve-se ter cuidado para antecedentes obstétricos de partos taquitócitos, partos prematuros em que não há trabalho de parto, dilatação cervical em que não há sinais de contrações, rotura prematura de membranas sem possuir causa, e abortamentos demorado com expulsão espontânea de membranas e feto sem contrações dolorosas (RODRIGUES LC, et al., 2003).

A ultrassonografia transvaginal é o exame aplicado ao diagnóstico de IIC durante o tempo gestacional, devido a sua sensibilidade e possibilidade de ter um diagnóstico mais precoce (FREITAS JRAO, et al., 2003). Já a ressonância magnética $(\mathrm{RM})$ vem sendo empregada desde a década de 90 como forma de avaliar as malformações fetais em gestantes e, com isso, consegue demonstrar excelente contraste tecidual e, assim, 
ganhar espaço nas doenças maternas na gestação, como o acretismo placentário, abdome agudo e neoplasias de pelve, com resultados verdadeiramente promissores (LEVINE D, 1997) .

O número de partos prematuros e a relação de dias de internação nas unidades de terapia intensiva (UTI), dias de utilização de antibióticos de amplo espectro e apoio psicológico às famílias que estavam interligadas ao processo acima resulta em alto custo para o sistema de saúde, tanto público como privado. Não obstante a isso, ainda não existem dados e estudo na literatura sobre o prognóstico de prematuridade em grávidas acometidas à cerclagem (HEDRIANA HL, 2008).

Ouzounian JG, et al. (2011) traz esclarecimento em relação à cerclagem cervical, sendo a cirurgia utilizada na tentativa de prolongar a gestação de mulheres com IIC. Existem três diferentes circunstâncias na forma como a cerclagem é realizada: a primeira é a eletiva, a qual, segundo o autor, é praticada em mulheres com diagnóstico de IIC, cuja definição se baseia no histórico de dilatação cervical sem esforço ou grande sofrimento, e, em sequência, por parto de feto vivo no segundo trimestre do período gestacional, entre 11 e 16 semanas (DEBBS RH, et al., 2009). Essas pacientes são classificadas como assintomáticas ao tempo da cerclagem cervical. Já a cerclagem de urgência ou terapêutica é o procedimento feito e acometido em gestantes assintomáticas que possuem alto risco, as quais são informados por ultrassonografia transvaginal que apresentam indícios de incompetência cervical, procedimento em que é incluído o comprimento cervical menor que $25 \mathrm{~mm}$ ou saliência de membranas ovulares no nível do orifício cervical interno, entre 16 e 24 semanas. Por fim, a cerclagem de emergência é uma etapa realizada em mulheres que apresentam dilatação cervical elevada ou igualada a 2 centímetros, apresentando membranas íntegras e sem indicação de trabalho de parto ou com visualização de membranas ovulares no nível do orifício cervical externo ou por meio dele entre 16 e 24 semanas (NELSON L, et al., 2009).

Sendo assim o objetivo do presente trabalho foi revisar artigos acerca do tema Incompetência istmocervical: cerclagem de emergência versus conduta expectante.

\section{REVISÃO BIBLIOGRÁFICA}

A cérvice uterina é composta por uma estrutura que tem a funcionalidade, durante a gestação, de primeiro conservar o concepto dentro do útero até a sua maturação e, seguinte a essa etapa, conseguir a dilatação suficientemente para conceder seu nascimento. São funções que precisam ser realizadas com frequência nas gestações seguintes. Durante o período gestacional, a cérvice uterina passa a ficar mais ativa, observando o ponto de vista bioquímico. No estado não gravídico, o colo do útero consiste, aproximadamente, em $80 \%$ de água e aumenta para $86 \%$ no final da gestação. Este aumento facilita a ação da elastina, que desestabiliza as fibras de colágeno (THOMSON A, et al., 1997). Ainda é ressaltado pelo mesmo autor que a concentração de colágeno abaixa em média de $30 \%$ a 50\% se houver a comparação ao período pré-gravídico, podendo, também, ocorrer mudança de componentes da matriz extracelular cervical.

Timmons B, et al. (2010) mostra em seu estudo que o amadurecimento da cervical deve ser constituído por fases distintas que se sobrepõem: 1) amolecimento isolado; 2) enfraquecimento (abrandamento com diminuição do comprimento); 3) dilatação em solução a contrações; 4) restabelecimento pós-parto.

\section{Principais características da incompetência istmo cervical}

Peixoto-Filho FM, et al. (2009) ressalta que a técnica da cerclagem foi iniciada há mais ou menos meio século, com o foco de evitar casos de prematuridade em pacientes com história clínica com predisposição de incompetência istmo-cervical. Ainda segundo relatos do mesmo autor, essa indicação era realizada no início, mas foi alterada com o passar do tempo. Alguns estudos propõem a cerclagem para gestantes que apresentem o colo curto detectado no momento da ultrassonografia e para pacientes que não apresentam história de incompetência istmo-cervical.

A incompetência istmo cervical é determinada pela inabilidade do colo uterino em sustentar-se fechado e reter o embrião até o termo, o que em muitos casos resulta em abortamento ou nascimento prematuro (ALVES APVD, 2016). A IIC caracteriza-se pela dilatação indolor aguda do colo do útero, atingindo a interrupção prematura de membranas, ficando, assim, responsável por de $10 \%$ a $25 \%$ das perdas recorrentes no segundo 
trimestre, assim como $10 \%$ dos partos prematuros e afetando $1 \%$ do total de pacientes grávidas (SHAMSHAD YM, 2008).

A IIC pode ser ocasionada por anormalidade traumática do colo uterino, acometido por curetagens, rupturas, em casos de cirurgias ou amputações do colo, irregularidades anatômicas, que podem ser por presença de tumores e miomas e, por fim, por anormalidades congênitas ou funcionais que se explicam pelas propriedades constitucionais do tecido conectivo do colo uterino. Tanto as normalidades congênitas como as traumáticas estão associadas ao aumento do risco de parto prematuro (NORMAN JE, 2007).

Fazer uma identificação precoce e garantida do trabalho de parto prematuro traz grande significância, pois, em muitos casos, pode ser fator de impedimento de nascimento de pré-termo ou em circunstâncias que 0 permitia acontecer em melhores condições. Os relatos anteriores são extremamente importantes como fator de risco para a repetição na gestação atual e, com a ciência, obtêm a aceitação, a fim de diminuir os agravos consecutivos da prematuridade, utilizando, assim, medidas para a prevenção no período do pré-natal, como avaliações higienodietéticas, uso de tocolíticos e corticoterapia (SALING E, et al., 2001).

\section{Quadro clínico e taxas de mulheres submetidas a cerclagem versus conduta expectante}

As gestantes com IIC, muitas vezes, já expõem um histórico de pressão pélvica, uma elevação quanto ao corrimento vaginal do tipo mucoso e uma expansão cervical indolente maior que $4-5 \mathrm{~cm}$. Sintomas estes que são acometidos em idades gestacionais, cada vez mais, de forma imatura em gestações seguidas (HARGER $\mathrm{JH}, 2002)$. A taxa de parto antes de 36 semanas nas gestantes submetidas à cerclagem profilática varia entre $20 \%$ e $25 \%$, nas cerclagens terapêuticas, $27 \%$ e $42 \%$, e nas de emergência, entre $53 \%$ e $77 \%$. A taxa de prematuridade nas gestantes submetidas a cerclagem profilática e terapêutica não é estatisticamente diferente, ao contrário da observada no grupo de gestantes submetidas a cerclagem de emergência (KHAN MJ, et al., 2012).

Kurup M e Goldkrand JW (1999), em um estudo americano, relataram que a sobrevida neonatal foi de $92,1 \%$ no grupo de cerclagem eletiva, $86,6 \%$ no grupo de cerclagem terapêutica e de $85,7 \%$ entre as gestantes submetidas à cerclagem de emergência. Referente aos dias de internação hospitalar, a média atingida foi de, de 6,2 dias $\pm 0,5$ dias, quando os valores foram para os recém-nascidos de mães submetidas à cerclagem profilática, 15,4 dias $\pm 8,8$ dias para as pacientes que passaram por cerclagem terapêutica e de 29,1 dias $\pm 8,2$ dias para os recém-nascidos de mães que se submeteram à cerclagem de emergência. No segundo trimestre, a perda gestacional acomete menos que 1\% das gestações identificadas (CULLEN S, 2017). Isso se dá por diversos fatores etiológicos, apesar disso, quando analisadas, percebe-se que cerca de metade destas perdas é acometida por causa idiopática e que, em meio aos fatores etiológicos agrupados para estas perdas, encontram-se a síndrome anti-fosfolípide, as contaminações genitais e a fragilidade cervical (MCNAMEE KM, et al., 2014).

Pereira L, et al. (2007), em um dos seus estudos retrospectivos, comparou a conduta expectante versus cerclagem em gestantes com cervicodilatação. As gestantes selecionadas apresentavam uma faixa de 14 semanas e 25 semanas e 6 dias atendidas, entre 1998 e 2005, em 10 centros (sendo quatro norteamericanos, dois tailandeses, um chileno, um finlandês, um irlandês e um indiano). A seleção foi específica para pacientes que apresentavam dilatação de $1 \mathrm{~cm}$ ou mais, tendo a somatória final de 152 participantes no grupo submetido à cerclagem e 73 no grupo submetido à conduta expectante. A idade gestacional para o diagnóstico foi expressivamente menor no grupo de cerclagem, com média de 19,1 semanas, em relação ao grupo expectante (mediana de 23,1 semanas). Já a dilatação cervical no diagnóstico foi paralela para os grupos, $1,8 \mathrm{~cm}$ no grupo cerclagem e 2,0cm no grupo expectante. Em análise aos desenlaces primários, foi obtido aumento expressivo em relação a prolongar a gestação (média de 12,4 versus 1,6 semanas, $p<0,001$ ) e a idade gestacional no parto (33 versus 25,9 semanas, $p=0,003$ ) no grupo cerclagem correlacionado com o grupo expectante. Não foi expressiva a diferença em relação à sobrevida neonatal entre os grupos $(66,2 \%$ versus $58,9 \%$, respectivamente).

Ciavattini A, et al. (2016), em um estudo conduzido na Itália, comparam grávidas acometidas à cerclagem de emergência ou à conduta expectante, no segundo trimestre de gestação. Nesse estudo, a inclusão das 
gestantes foi entre 14 e 24 semanas e com dilatação cervical igual ou de elevação de $1 \mathrm{~cm}$. Todas as pacientes aceitaram a realização do procedimento e compuseram o grupo cerclagem 18 gestantes, as que se recusaram fizeram parte do grupo de controle composto por 19 gestantes. A comparação dos grupos foi em relação à quantidade de dilatação cervical $(3,6 \pm 1,6 \mathrm{~cm}$ no grupo cerclagem e 4,2 $\pm 2,0 \mathrm{~cm}$ no grupo controle) e por comprimento do colo (10,4 $\pm 5,3 \mathrm{~mm}$ no grupo cerclagem e 13,1 $\pm 10,5 \mathrm{~mm}$ no grupo controle). Sobre os desfechos fundamentais, os autores obtiveram os resultados para os grupos cerclagem e controle, descritos para: idade gestacional no parto $34,8 \pm 6,7$ versus $26,7 \pm 7,4$ semanas, tempo entre o diagnóstico e o parto $16,8 \pm 7,9$ versus $7,2 \pm 6,5$ semanas, e peso do recém-nascido $2.814 \pm 859$ versus $1.482 \pm 1.172$ gramas, apresentando diferença significativa para as três variáveis.

\section{Tipos de cerclagem}

A cerclagem pode ser obtida através da abordagem vaginal ou abdominal. Alguns estudos anteriores indicam que existe benefício quando relacionada ao tratamento cirúrgico, haja vista que gestantes apresentam maior tempo de incubação até o parto, maior idade gestacional ao nascimento e diminuição da taxa de prematuridade (EHSANIPOOR RM, et al., 2015).

A cerclagem cervical é o procedimento cirúrgico empregado na tentativa de estender tempo de gestação de mulheres com IIC e que têm presença maior que $2 \%$ em todas as gestações (OUZOUNIAN JG, et al., 2011). Segundo Gomes ALFM, et al. (2012), o tratamento cirúrgico por meio da cerclagem do istmo cervical é o padrão ouro, e a abordagem para a realização dela é a via vaginal. Ainda segundo o autor, o período ideal está entre 12 e 16 semanas de gestação, enfatizando que com o repouso no leito pode-se obter resultados semelhantes à cerclagem no tratamento das perdas gestacionais de repetição.

A cerclagem de emergência proporciona aumento de taxas de prematuridade, desta forma consegue gerar maiores complicações quando correlacionada com a cerclagem profilática ou terapêutica (KHAN MJ, et al., 2012). É notável que os resultados neonatais apresentam mais desfavor na cerclagem de emergência, da mesma forma quando avaliados especificamente recém-nascidos com baixo peso, recomendando que exista influência de processos inflamatórios subclínicos presentes nesses resultados (KUON RJ, et al., 2015).

\section{Exames}

A utilização da ultrassonografia transabdominal (USG TA) e, logo depois, da ultrassonografia transvaginal (USG TV) como forma de melhor visualizar o colo do útero, trouxe melhor forma de compreensão da IIC. Em contrapartida, em grande parte dos estudos publicados mostraram-se resultados inconsistentes à medida que são executados com número inferior de gestantes, que estão submetidas a riscos diferentes para prematuridade e causas diferentes de IIC (BORGHI TF, 2016).

Um estudo realizado por Carvalho MH (2010), por método retrospectivo, avaliou 131 gestantes, com primeira gestação, que foram submetidas à cerclagem profilática. As considerações por meio da ultrassonografia foram realizadas nos instantes anterior e posterior à cerclagem em meados de 20 e 24 semanas. O momento de constatar o afunilamento cervical foi determinado com a dilatação do orifício interno do colo uterino, cuja medida do seu diâmetro anteroposterior era maior que $5 \mathrm{~mm}$. As dimensões do afunilamento cervical pós-cerclagem resultaram em diferença significativa entre as gestantes com parto < 32 semanas (média de $26,7 \mathrm{~mm} \pm 6,44$ ) e as gestantes com parto $\geq 32$ semanas (média de $5,8 \mathrm{~mm} \pm 2,22$ ) $\mathrm{p}=0,036$. Entretanto, quando $\mathrm{o}$ afunilamento cervical foi observado como variável categórica, não apresentou diferença significativa em correspondência a IG parto < 32 semanas ou IG parto $<34$ semanas (CARVALHO MH, 2010).

A RM de colo uterino (RMCU), em um alguns poucos estudos e publicações, demonstrou uma aparência única, com aspectos morfológicos específicos, o que conseguiu representar o acesso à microestrutura do colo, por meio da intensidade de sinal (hidratação), espectroscopia (metabólitos), mais adiante uma avaliação concisa da biometria e da zona estromal bem individualizadas (BRANDAO RS, et al., 2008). A RM tem evidenciado superioridade quando comparada à US, em questão de resolução e de contraste tecidual (HOUSE M, et al., 2005), ainda assim, segundo o mesmo autor, é um método favorável na avaliação da zona estromal, auxiliando na compreensão das alterações fisiológicas e não fisiológicas do colo do útero 
gestacional . É o único exame que consegue prover informações sobre o estroma cervical, admitindo um maior entendimento das modificações fisiológicas da cérvice (RAE DW, et al., 2001).

Mattar R, et al. (1997) já afirmava que a importância da IIC reside, principalmente, no fato de que, com o tratamento cirúrgico, os resultados são, amiúde, favoráveis, permitindo que uma mulher anteriormente infértil passe a ter gestações com viabilidade fetal. E, de maneira geral, a cerclagem amplia o risco de infecções puerperais $(6 \%)$, internações hospitalares e realização de cesarianas. As complicações mais comuns são: lacerações cervicais (3-4\%), estenose cervical (1\%), hemorragia ocasionadas por lesão dos vasos uterinos, deslize de sutura, transfixação da bexiga, necrose da parede vaginal anterior ou posterior, amputações de colo, esterilidade secundária e fístula vésico-vaginal (ROZAS A, et al., 2003). Nomura ML (2003) diz que, independentemente do exame de ultrassonografia usado como dispositivo para estabelecer diagnóstico, a utilização do toque vaginal ainda é indicada como método de rastreio e seleção das pacientes para propedêutica de auxílio.

Uma vantagem na realização do diagnóstico de insuficiência cervical, apesar da ausência de um teste objetivo, é a possibilidade de realização da cerclagem. Para tal, se faz necessário o correto diagnóstico e planejamento terapêutico para futuras gestações (BROWN R, et al., 2013). Segundo Nomura ML (2000), é preciso dar importância para a obtenção de uma anamnese bem específica e detalhada, pois o ponto do diagnóstico pode estar no histórico de antecedentes obstétricos, e, segundo o mesmo autor, o diagnóstico certo ainda é complexo e difícil de obter, principalmente durante a gestação, haja visto que fatores de risco para parto prematuro têm um baixo valor preditivo.

\section{CONSIDERAÇÕES FINAIS}

O diagnóstico de incompetência cervical embasa-se, primariamente, na história clínica, podendo ser efetuado antes ou durante a gestação. A cerclagem de emergência constitui-se perante dilatação do colo uterino, e o tratamento da IIC deve ser obtido de preferência próximo a 14ª semana da gravidez, quando ainda não houve modificações cervicais e já ultrapassou o tempo em que ocorrem abortos precoces. A cerclagem é a conduta com maior aceitabilidade de acordo com a literatura, devido aos índices de sobrevivência fetal e das gestantes portadoras de IIC no período que antecede o parto e após passar pelo tratamento e procedimento cirúrgico.

\section{REFERÊNCIAS}

1. ALVES APVD. Associação entre fatores genéticos e risco aumentado de prematuridade em pacientes com antecedente de incompetência cervical. 2016.

2. AMERICAN COLLEGE OF OBSTETRICS AND GYNECOLOGIST. ACOG practice bulletin. Cervical insufficiency. Int J Gynaecol Obstet. 2004;85(1):81-9.

3. BORGHI TF.Avaliação do colo uterino de gestantes com incompetência istmo-cervical por meio da ultrassonografia transvaginal bidimensional e tridimensional / Thais da Fonseca Borghi. -- São Paulo, 2016.

4. BRANDAO RS, et al. Avaliação biométrica do colo uterino durante a gestação por meio da ultrassonografia transvaginal e Ressonância Magnética. Radiol Bras. 2008; 1(4):235-39.

5. BRASIL. Ministério da Saúde. Gestação de alto risco: manual técnico. 5. ed. Brasília: Editora do Ministério da Saúde, 2010.

6. BROWN R, et al. Cervical insufficiency and cervical cerclage. J Obstet Gynaecol Can. 2013;35(12):1115-27.

7. CARVALHO MH. Avaliação dos fatores preditivos para prematuridade espontânea em gestantes submetidas à cerclagem cervical eletiva. São Paulo: Universidade de São Paulo;2010.

8. CIAVATTINI A, et al. Effectiveness of emergency cerclage in cervical insufficiency. J Matern Fetal Neonatal Med. 2016;29(13):2088-92.

9. CULLEN S, et al. An exploration of the prevalence and patterns of care for women presenting with mid-trimester loss. Ir J Med Sci.2017;186(2):381-6.

10. DEBBS RH, et al Contemporary use of cerclage in pregnancy. Clin Obstet Gynecol.2009;52(4):597-610.

11. EHSANIPOOR RM, et al. Physical examination-indicated cerclage a systematic review and meta-analysis. Obstet Gynecol.2015;126(1):125-35. 
12. FREITAS JRAO,et al. Evolução do comprimento cervical uterino na gestação, avaliado pela ultra-sonografia transvaginal. Rev Bras Ginecol Obstet. 2003; 25(2):115-21

13. GOMES ALFM, et al. Incompetência istmo-cervical: atualização. Rev Med Minas Gerais 2012; 22 (Supl 5): S67-S70.

14. GOULDING E, LIM B. McDonald transvaginal cervical cerclage since 1957: from its roots in Australia into worldwide contemporary practice.BJOG.2014;121(9):1107.

15. HARGER JH. Cerclage and cervical insufficiency: an evidence-based analysis. Obstet Gynecol.2002;100(6):1313-27.

16. HEDRIANA HL, et al. Is there value for serial ultrasonographic assessment of cervical lengths after a cerclage? Am J Obstet Gynecol. 2008;198(6):705.e1-6.

17. HOUSE M, et al. Magnetic resonance imaging of the cervix during pregnancy: effect of gestational age and prior vaginal birth. Am J Obstet Gynecol. 2005 Oct;193(4):1554-60.

18. KHAN MJ, et al. Evaluation of outcomes associated with placement of elective, urgent, and emergency cerclage. J Obstet Gynaecol India. 2012;62(6):660-4..

19. KUON RJ, et al. Impaired neonatal outcome after emergency cerclage adds controversy to prolongation of pregnancy. Plos One.2015;10(6):e0129104.

20. KURUP M, GOLDKRAND JW. Cervical incompetence: elective, emergent, or urgent cerclage. Am J Obstet Gynecol.1999;181(2):240-6.

21. LEVINE D, et al. Placenta accreta: evaluation with color Doppler US, power Doppler US, and MR imaging. Radiology. $1997 ; 205(3): 773-6$.

22. MATTAR R, et al. Assistência à insuficiência istmocervical. Femina 1997; 25:51-3.

23. MATTAR R, et al.Further experiences with the Shirodkar operation. Am J Assistência à insuficiência istmocervical.; Obstet Gynecol 1999; 85:792. 25:51-3.

24. MCNAMEE KM, et al. Mid-trimester pregnancyloss. Obstet Gynecol Clin North Am.2014;41(1):87-102.

25. NELSON L, et al. Pregnancy outcomes following placement of elective, urgent and emergent cerclage.JMatern-Fetal Neonatal Med.2009;22(3):269-73.

26. NOMURA ML, Passini Júnior R, Silva JCG, Vaz MS, Amaral E, Pereira BG. Resultados gestacionais e perinatais de gestações com insuficiência cervical submetidas a cerclagem eletiva. Rev Bras Ginecol Obstet. 2003; 25(7):483-9

27. NOMURA ML. Resultados gestacionais de mulheres submetidas a cerclagem do colo uterino durante a gravidez. Campinas, S.P.: [s.n.], 2000

28. NORMAN JE. Preterm labour. Cervical function and prematurity. Best Pract Res Clin ObstetGynaecol.2007;21(5):791806.

29. OUZOUNIAN JG, et al. Cervical length and delivery outcomes in patients with prophylactic cervical cerclage. Am J Perinatol. 2011;28(4):273-6.

30. PEIXOTO-FILHO FM, Oliveira JF, Porto MFS. O papel da cerclagem nas gestações múltiplas: revisão sistemática. FEMINA, outubro 2009, vol 37, no 10.

31. PEREIRA L, et al. Expectant management compared with physical examination-indicated cerclage (EM-PEC) in selected women with a dilated cervix at 14(0/7)-25(6/7) weeks: results from the EM-PEC international cohort study. Am J Obstet Gynecol.2007;197(5):483.e1-8.

32. RAE DW, et al. Magnetic resonance imaging of the human cervix: a study of the effects of prostaglandins in the first trimester. Hum Reprod. 2001 Aug;16(8):1744-7.

33. RODRIGUES LC,et al. Caracterização da gravidez com insuficiência istmocervical. Rev Bras Ginecol Obstet. 2003; 25(1):29-34.

34. ROZAS A. Incopetência Cervical. Rev Fac Ciênc Méd. 2003; 5(2):1-9.

35. SALING E, et al. A simple, efficient and inexpensive program for preventing prematury. J Perinat Med 2001;29:199211.

36. SHAMSHAD YM, et al.Evaluation of cervical cerclage for sonographically incompetent cervix in at high risk patients. J Ayub Med Coll Abbottabad. 2008;20(2):31-4.

37. THOMSON AJ, et al. The Cervix. In: Elder MG, Lamont RF, Romero R, editors. Preterm labor. United States of America: Churchill Livingstone; 1997. p.445-55.

38. TIMMONS B, et al. Cervical remodeling during pregnancy and parturition. Trends Endocrinol Metab.2010;21(6):35361 